\title{
Identifying Cancer in a Woman with a Breast Implant Following Prior Mastectomy for Breast Cancer
}

\author{
Richard M Fleming'*, Matthew R Fleming ${ }^{1}$, Tapan K Chaudhuri ${ }^{2}$ and William C Dooley ${ }^{3}$ \\ ${ }^{1}$ FHHI-OmnificImaging-Camelot, Los Angeles, CA, USA \\ ${ }^{2}$ Eastern Virginia Medical School, Norfolk, VA, USA \\ ${ }^{3}$ Oklahoma University Health Science Center, Oklahoma City, Oklahoma, USA \\ *Corresponding Author: Richard M Fleming, FHHI-OmnificImaging-Camelot, Los Angeles, CA, USA.
}

Received: October 24, 2019; Published: November 13, 2019

DOI: $10.31080 /$ ASPS.2019.03.0436

\begin{abstract}
Breast implants are associated with an increased risk of developing inflammatory reactions - both within the breast and systemically - as well as cancer. It is not uncommon for women who have undergone mastectomy or partial mastectomy for the removal of a prior cancer, to undergo breast implants. Measurement of regional blood flow (RBF) and metabolic differences using FMTVDM* differentiate tissue types and can be used in women with dense breasts and breast implants - including those with prior surgical intervention for the treatment of breast cancer - to determine if inflammation, precancerous changes or breast cancer is present. We present once such case - evaluated by FMTVDM* - of a woman with breast implants placed after prior surgery for breast cancer.
\end{abstract}

Keywords: FMTVDM; Breast Cancer; Mastectomy; Breast Implant

\section{Introduction}

The risk of inflammatory changes, potential autoimmune diseases and breast cancer in women with breast implants is now well recognized [1-6]. Given the prevalence of breast implants and the likelihood that breast implants will not become a thing of the past, it is important that clinicians be able to monitor the overall health of the women and men with breast implants by measuring changes in breast health [7-9] on a Health-Spectrum as shown in figure 1. One of the common reasons for breast implants are among women who have previously undergone surgical removal of part or all of a breast for the treatment of breast cancer. Such women must be closely monitored for possible recurrence or the development of a new secondary cancer.

We present a case of a woman with both dense breast and breast implants that noticed breast irregularities and requested further measurement of metabolic and regional blood flow (RBF) differences using FMTVDM.

\section{Patient study**}

Following standardized preparation and introduction to the nuclear imaging laboratory, and following the signing of informed consent per laboratory protocol, a middle aged Caucasian woman
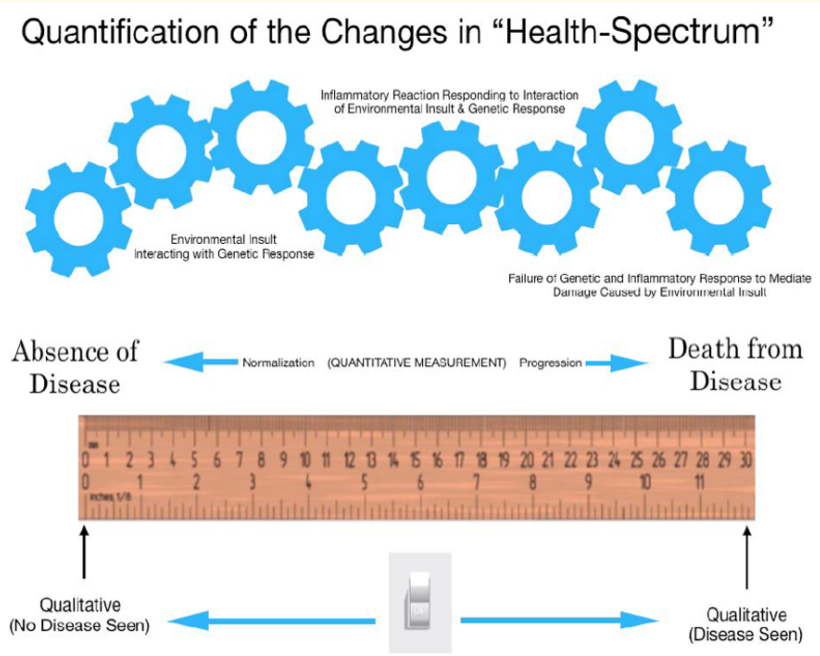

Figure 1: Progression or regression of health across a health-spectrum is a measureable continuum of transitional changes occurring over time resulting from the interaction of the specific person's cellular genetic composition and the environmental influences impacting specific cells and tissue [9]. 
presented for evaluation using FMTVDM [7-9]. She had previously undergone partial mastectomy of the left breast for cancer and had not noted any breast irregularities. She had never smoked nor previously taken hormone therapy.

The result of her FMTVDM is shown in Figure 2, where several regions of interest (ROIs) are presented along with FMTVDM measurements for regional blood flow (RBF) and metabolism. The majority of the FMTVDM counts are within the expected range for normal breast tissue; however, in the upper breast tissue were two regions that turned out to be breast cancer with values of 386 and 396 [7-9].

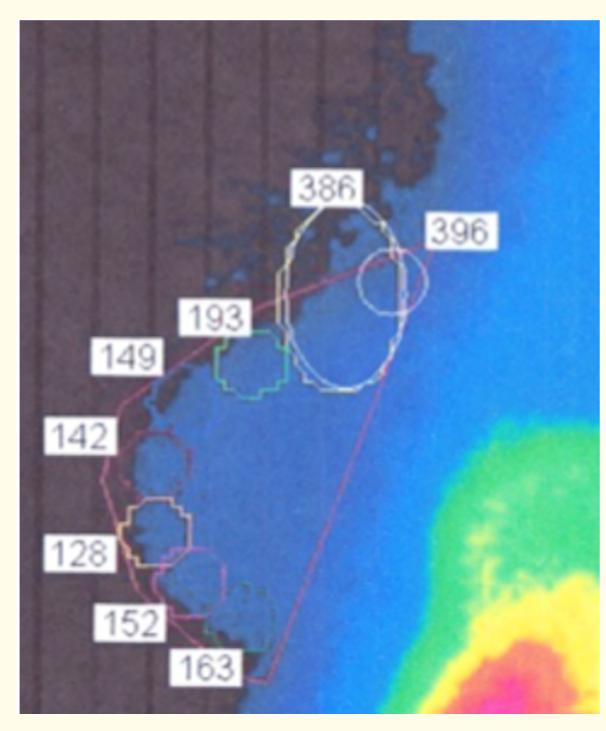

Figure 2: FMTVDM results for a woman with cancerous breast tissue following prior mastectomy and breast implant.

\section{Discussion}

Recurrence of breast cancer is a constant concern both for the patient and the clinician following the patient. The introduction of a breast implant complicates the ability to find recurrent or new cancers, while at the same time, the implant itself may result in inflammatory or cancerous changes that need to be considered.

Measurement of RBF and metabolic changes allow measurements in women, and men, who have undergone mastectomy, breast implants, have dense or normal breast tissue. By making such measurements, new and recurrent breast cancers can be found without the limitations of mammography.

\section{Conclusion}

FMTVDM provides the only diagnostic tool, which can measure changes in RBF and metabolic differences. Such measurements can differentiate tissue even when prior surgical intervention has complicated the appearance of the breast tissue - including mastectomy and breast implants.
*FMTVDM = The Fleming Method for Tissue and Vascular Differentiation and Metabolism.

** FMTVDM provided to patient following standard laboratory explanation and signing of informed consent.

\section{Acknowledgments}

FMTVDM is a utility patent issued to first author. All figures reproduced with expressed consent of first author. The authors wish to thank David L Prothro, MD of Nevada Arrhythmia Services Inc. in Reno, Nevada for independently conducting patient studies and providing results. The authors also wish to thank all the women and men with dense breasts and to Joe and Nancy Cappello, PhD.

\section{Bibliography}

1. Bizjak M., et al. "Silicone implants and lymphoma: The role of inflammation". Journal of Autoimmunity 65 (2015): 64-73.

2. De Boer M., et al. "Is explanation of silicone breast implants useful in patients with complaints?" Immunology Research 65 (2017): 25-36.

3. Orciani M., et al. "Inflammation by Breast Implants and Adenocarcinoma: Not Always a Bad Company". Clinical Breast Cancer 17.4 (2017): 286-292.

4. Cohen Tervaert JW., et al. "Silicone implants and autoimmune rheumatic diseases: myth or reality". Current Opinion on Rheumatology 29.4 (2017): 348-354.

5. Leberfinger AN., et al. "Breast Implant-Associated Anaplastic Large Cell Lymphoma: A Systematic Review". JAMA Surgery 152.12 (2017): 1161-1168.

6. Collett DJ., et al. "Current Risk Estimate of Breast Implant-Associated Anaplastic Large Cell Lymphoma in Textured Breast Implants". Plastic and Reconstructive Surgery 143 (2019): 30s40S.

7. The Fleming Method for Tissue and Vascular Differentiation and Metabolism (FMTVDM) using same state single or sequential quantification comparisons.

8. Fleming RM and Dooley WC. "Breast enhanced scintigraphy testing distinguishes between normal, inflammatory breast changes, and breast cancer: a prospective analysis and comparison with mammography". Integral Cancer therapy 1.3 (2002): 238-245.

9. Fleming RM and Fleming MR. "The Importance of Thinking about and Quantifying Disease like Cancer and Heart Disease on a "Health-Spectrum" Continuum". Journal of Comprehensive Cancer Research 3.1 (2019): 1-3.

\section{Volume 3 Issue 12 December 2019 (C) All rights are reserved by Richard M Fleming., et al.}

